\title{
CYANOPHYCEAEN AND CHLOROPHYCEAEN FLORA OF SIKKIM HIMALAYAS, INDIA
}

\author{
Satendra Kumar, M.K. Suseela and Kiran Toppo \\ Phycology Section \\ Natismal Botanical Rescarch Institute, Lucknow - 226001, India
}

\begin{abstract}
The prewent paper enumerates 30 laxa of fresh water algae, in which 18 helong to de chass

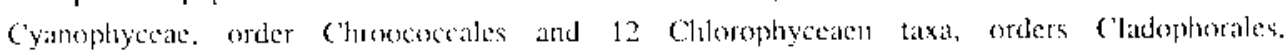

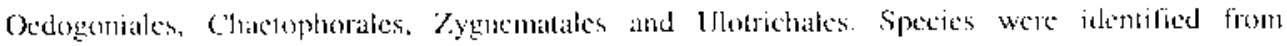
collected samples of high altitudinal anses of Sikim Jimalayas. All taxal lave treen reponted for the litst time from the study area.
\end{abstract}

key words: Cyanophyceas, Chloroplyecac, Sikkim Jimalayas.

\section{INTRODUCTION}

The study of liesh water aly:al flora of high allitudinal zames of India has not been given due attention. A lew records of Allied (1978) from

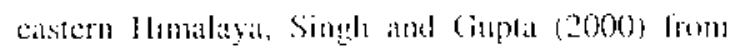

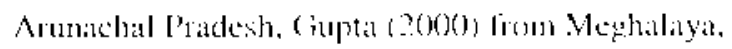
Khate and Susecta $(2004)$ fiom Ittaranchal gave

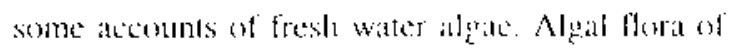
Sikkim state has not been well studied, except some cursery fepurte of Santra (1984), Pratiald and Mista (1987) from (ianglok and its sumounding

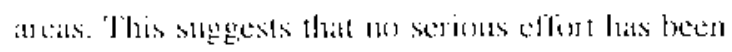
mate to explene the aleal thera of Sikiom flimislityans.

Sikim state due lo its proximity to Himalayals filces extrene climatio emations. On an average the: maximun kemperature reserds gote and minimum 12\%(. Ritinfall (x)turs thromghout the year and stalle ats as whole gets $80-90 \%$ of the

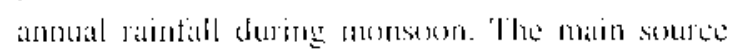
of Hate in small walcer bodies like probls, ditches. streams are from water falls and glaciers. Fresh water algal thora of different altitudinal ranges (1300-1700 m) of Sikkm has been surveyed.

\section{MATLRIALS AND METHODS}

Samples were collected from probls, ditches, splashed rocks, slow-running streams. water falls and moist sosils of three distimed places witl dillement allitudinal binges: Vandil. $1525 \mathrm{~m}$ : Gangtuk, $1700 \mathrm{~m}$ and Rumtek Motriatry, $1700 \mathrm{~m}$ during the year 2000 and 2001 . These samplese were preserved in $10 \%$ tinmaline and depensited at Plyycologe laboratory of National Bentanical

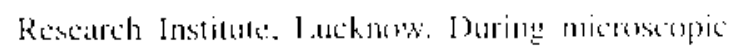
observations micos photegraptas were lakest in

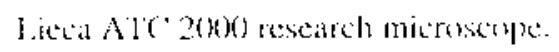

The identilication of taxal were dene by listowing standard publicaltionts: Prencont (1951), Tiffiny and Brith (1952). Desikindatry (1920), Randillawa (1959), Anand (1998), Kilnt and (Buptat (1998). The species arranged all phatketically with lokality and sample numbers given in patenthesis.

\section{SPRCIES ENUMLRATION}

Class: Cyanoplycteae

Order: Chroococcales

Fanily: Chroococcaceate 
Anacystis rupestris (Lyng.) Drouet and Daily Pl. 1. Fig. 10

(Tiffary and Britum 1952, p. 331. Pl. 89, Fig. 1039)

Colls cylindriatl, free fluating, 6.2 , $1 \mathrm{~mm}$ in dameter, cell content blue green, homogeneous.

Locality: Small water stream at Namchi lowards Namthinge and Silignet.

Sample No.: NBRR. ALSH

1phanothece rastagnei (Kuty) Rahesh P. 1. lïgs. 14 and 20

(1)esihathary 1959, p. 106, J']. 26, Fig. 1.3)

The sedts atre aval to cylinderical. $3.6+\mathrm{km}$ in cliameter and 6.8 fum long sarrounded by thick mucilaginus sleath, the cell content blus green and loungenesus.

Locality: Simall water stecam al Natuchi howards Niumhang: and Silicruri.

Sample No.: N13RI. AL.SI (1)

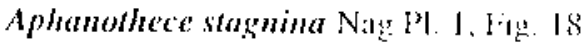

(Desikatchas y 1959, p. 142, 11. 22. liges. 4.5 and 9)

Thallus sonall, gredatinous, colls longer thatu broud,

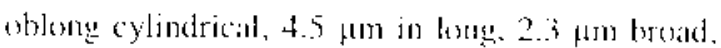
cell conten blue greem, bumbengents.

Iocality: A smbalt wales tall al Candlli market in (ianetok.

Sample No.: NBRI. AI.S! I 023.

Chroococas minimus (Keiss.) I.cmm P'. 1. Figs. 12 and 19

(1)sikachary 1959, [. 106, P1. 26, Hig. 1.3)

Colonies 2-8 cells, enclosed by thin mudatginous sheith, each cell spherical to cllipsoidal with 4.5 frm in diameter.

Locality: From staguant water at the road side, Giangtok.

Sample No.: NBRI. AI.SH-08.

\section{Chroococcas minor (Kulz.) Nag Pl. I, Fig. I}

(Desikachary 1959. p. 105. Pl. 24, Fig. I)

Cells spherical in pars, 4 fam in diameter. enclosed by thin mucilagimous sheath. cell content blue green. homogencous.

Locality: On stagnant water at the rond side. (iatuglok.

\section{Sample No.: NBRI. NI.SII-0S.}

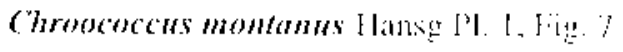

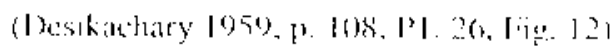

Thallus muciliginous. cells bemi-sphetical in shalpe, $\$ .2$ pom in diameerer. enclosed hy thin musilaginus shealta the cell wall thin and suruolth.

Locality: Sigueced firm sulumetged plats hedow the watce lall tomatrds [aummanu [uk.

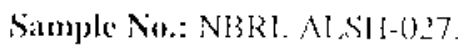

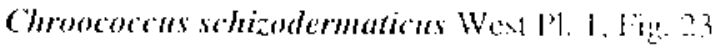

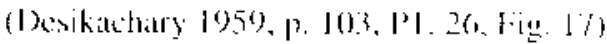

(cells in proups of 2-4, owat in shape, 7.2 pun in

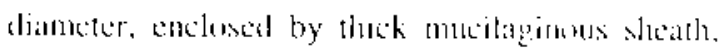
lamellited, cell content blac gresen witl smenth ce!l wiall.

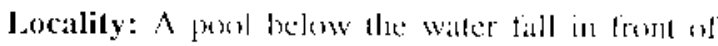
Rumtek Moritstry.

Simple No.: NIBRI. ALSHL-(14)

Chroococeus sarius Braun P]. 1, [ن̈gs. 16 and 24

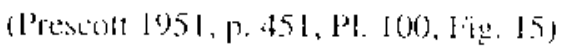

An iscengular shaped colony of $2-8$ spherical cells enclused by thick hyaline mucilaginums sheath, cell content blut green, homogeneous, cells 2.8 fm in ditmeter.

Locality: lirom stagnant water at the noad side, Gangtok.

Sample No.: NBRl. ALSII-08. 
Glococapsa atrata (Turp) Kutz Pl. 1, Fig. 5

(Desikilchary 1959, p, 139, P1, 27, Fig. 6)

Thallus mucilaginus with $2-4$ cells, spherical, surrounded by thin mucilaginous sheatb. $4.5 \mathrm{~km}$ in diameter. eell content hlue green, homogeneous.

locality: Small water strearm al Namelsi towards Nanthande and Siligguri

\section{Sample No.: NiBRI. NI.SH I 88 .}

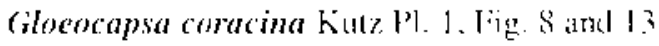

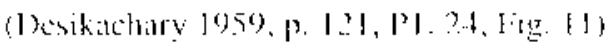

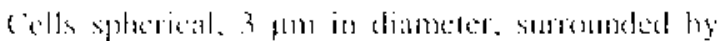

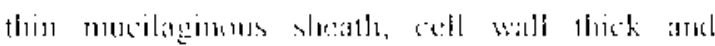
srmesolth.

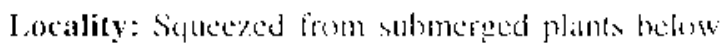

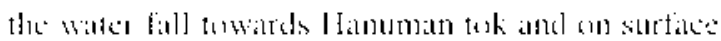

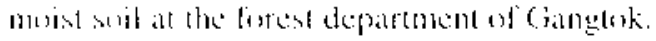

Simple Niss.: VIrRT. AI.SII-(3) 3, 027.

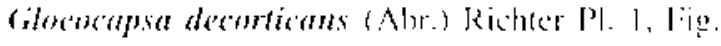
4

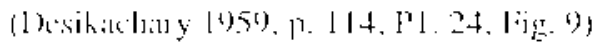

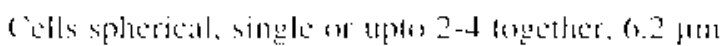

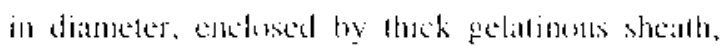

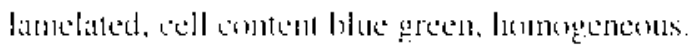

Locality: A roint side Hatter tank all (bangeloh and

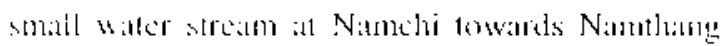
itnd Silinguri.

Sample Nos.: VH3lil. Alsli-03,0188.

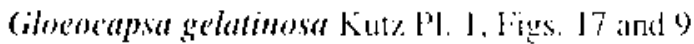

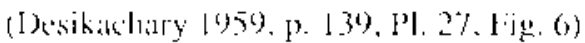

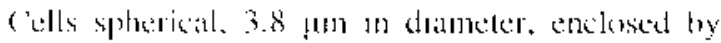

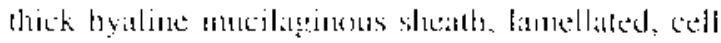
comtent hlus geren, homogencous.

I,ocality: Small water stream at Nombhi towards Ninuthang and Siliguri.

Sample Yo.: NBRI. NI.SH-0188.

Glococapsa montana Kutz I'1, 1, Migs, 2, 3, 15 and s.

ECOPRINT VOL 12, 2005
(Desikachary 1959, p. 123, P1. 24. Fig. 14)

Cells spherical, $5.2 \mu \mathrm{m}$ in diameler. single or two together in a colony, enclosed by thick byaline mucilaginous sheath, lamellated. cell content blue green, homkgencous.

Locality: Small watef stream at Namehi tonvartes Namthang and Siligurs and an stagname water of

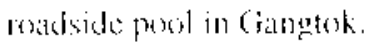

Sample Nos. NIBRI. AI.SII (R, OISB

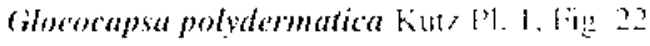

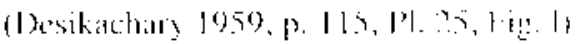

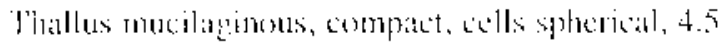

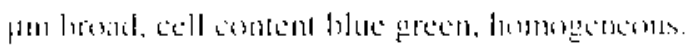

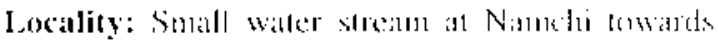

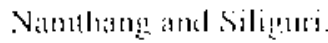

Simple No.: NBRRI. AL.SII- t\}ISS.

Gococapsa kuetingiama Nign l’1. 1, Yig. I1

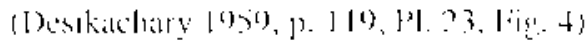

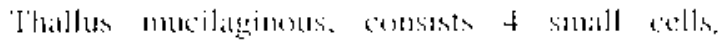

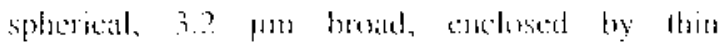

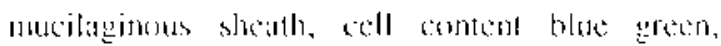
boumbertencens.

locality: Small water striam at Namehi fowyads Nomulamer and Siliguri.

Sumple No.: NBRI. AISII OJ

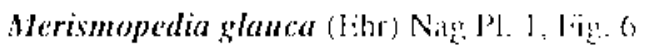

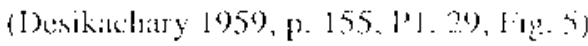

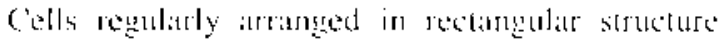
wijth crenate margin, cells while, hemisphericil, 2 2.3 flm in diameter, cell content blue green. homegenedus.

Locality: On rocks with splashed walter near water fall in front of Rumtek Monastry. 
Sample No.: NBRI. ALSH-049.

\section{Merismopedia elegans A, Br Pl. 1, Fiu. 25}

(Desikachary 1959, p. 150, P1. 29. Fig. 9)

Colomics small with 16 celled. cells oblong. chosely arranged having 5.5-6, $\mu$ mm in diameter, lree fluating.

Locality: On rorhs splasiled by walter an Rumtek Momlastry.

\section{Sample No.: NIBRI. AISH1-(0).31}

Misrocystis bengalensis Bemerij IP. I, Iijg. 20

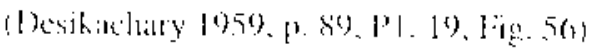

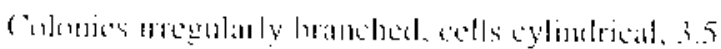
!em in dianeter, shealla distinct, surrounds the number of datughere conlonies.

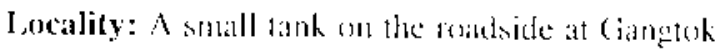
view pritut.

Sinuple No.: NBBRI. AISII-0) l3

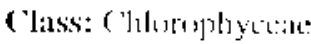

Oroler: C lakdopturalles

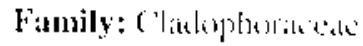

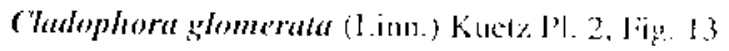
(Tillany and Britton, 1952, p. 45, I'1. 1.3, I ijg. 93)

(atls of main axis $205.2 \mu$ m loug, 1.5 .8 fum hroat and bataching in Y shaped structure, $11.5 \mu \mathrm{m} \mathrm{Jomg}$ 12.2 man hrond often a glomerate clusters with reticulate chloroplast.

Locality: $A$ small pend at the (Gangtek view point.

Simple No.: NBRI. Al.SH10106.

Cladfthora lactevirens (i)ilw.) Kuetz P. 2,

I.ip. 6

(Prasald and Misra 1992, p. 54, Pl. 7, Fig. 6)

Epilithic, branching of the filament pseudodichotomous, cells of main axts $315 \mu \mathrm{m}$ long, 60 utm in diameter, cells of branchlets $50.6 \mu \mathrm{m}$ long. $25.2 \mu \mathrm{m}$ in dianeter.

Locality: On rocks in the stream at Namchi.

Sample No.: NBRI. AI.SH-092.

Order: Oedogoniales

Family: Oedogoniaceile

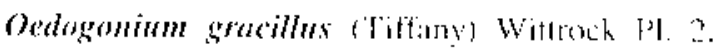
Iiㅣㅇ. I0

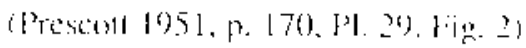

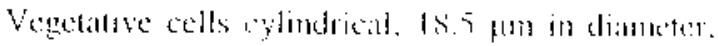

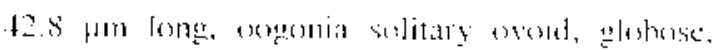

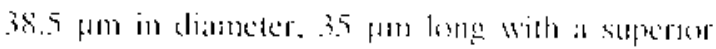

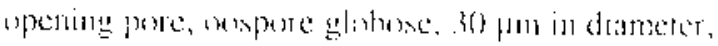
billings the ategromium.

locality: On cochs splisbed hy watce at Namkdi.

Sample No.: NislRI. AI.SHI-6I9?.

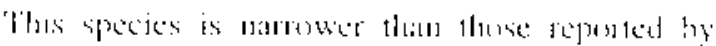

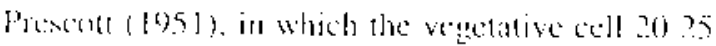

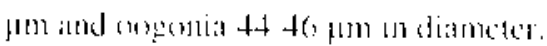

Otdegoniam sabatrokatam Tifling and Bulloul ly.

2. lijgs 5 aldul 1 ?

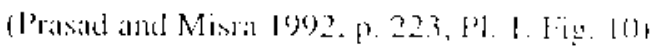

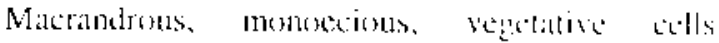

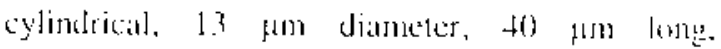

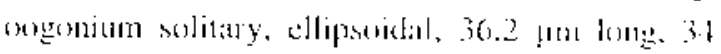

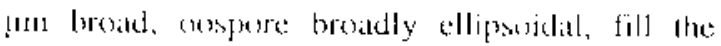

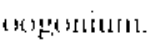

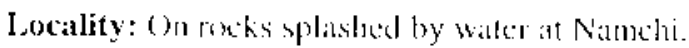

Sample No.: NiBRI. ALSH I-0192.

Oedogoniam varians Wittr et L.und Pl. 2. Figg. 3

(Prasiad and Misra 1992, p. 73, PJ. 11, Fig. (1)

Vegetative cells eylindricitl, $13.5 \mathrm{fm}$ in diameter. 50-55 $\mathrm{mm}$ long, oogonia solitary, depressed,

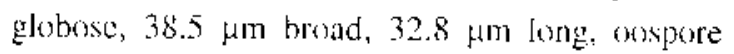
globose and $29.5 \mu \mathrm{m}$ in diameter, till the ogonium.

Locality: A smal] pool at the Gangtok view point. Sample No.: NBRI. ALSH-0106. 
Order: Chatophorales

Family: Chatophoraceac

Stigeoclonim tenue (Agar.) Kuetz P1, 2. Fig, 1

Kant and Ciupta 1998. p. 109. PI. 108. Fig. 3)

!ialancint dichotomously hranched, branches

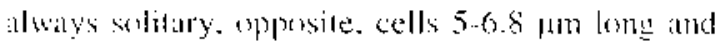
7.2 .

Iacality: Small stream on the way of catcostok v心 puint.

Siample No.: VRRT. NI.SII-0)]4S.

Order: 7ygnematales

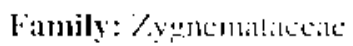

Spirogyra exils W. เ. W.S. West Pl. 2. Jig.

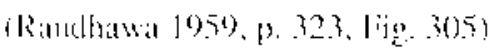

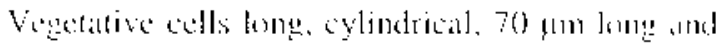

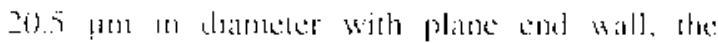

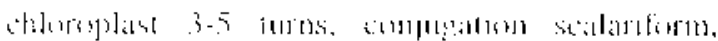

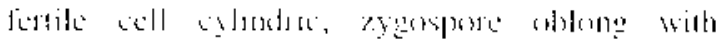

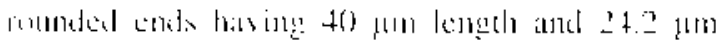
malche.

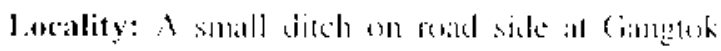
lavi siluml.

\section{Simple: No.: NIBRI. NISHL-(0I?).}

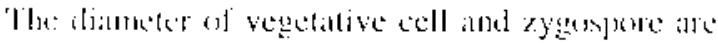

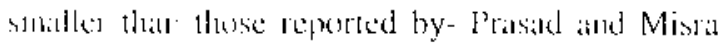

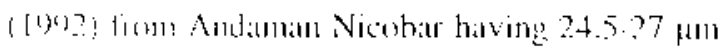

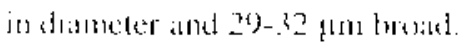

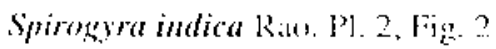

(Ankmd 1948, p. 5.t. Fig. 167)

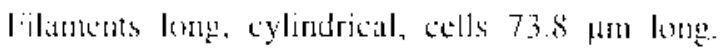
22.5 fun in diameter with plane end will chatomulass single, solititry, spirally amanged in ribbon like stondure with $2-3$ turns, conjugation by

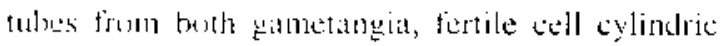
(x)me times slinblly swollen), zygospores spherical, latterally compressed with $32.5 \mu \mathrm{m}$ in diameter.

Locality: A sirall dith on rout side at Gianglok tiaxi stand.

Sample No.: NiBRI. ALSII-0129.
Spirogyra pratensis Transeau P1. 2, V̈gू. 9

(Prasad and Misra 1992, p.87, P1. 14. Figs. 5.7)

Vegetative cells $18.2 \mu \mathrm{m}$ in diameter, 60 !m 1 แnng with plane end wals having sinț spe spiral chloroplast making 2-3 turns, conjuation scalartform. cubes formed by botls gametingiat. fertile cell fusiform intlated upur 34.5 !n in

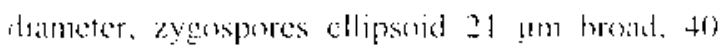
tam lomg!

Locality: In the water stleam mear wather fiall towards Jannuman iok.

Sample No.: NBRI. AI.SHI-(0)?

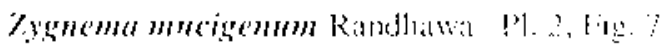

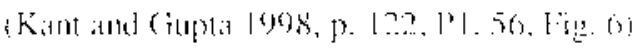

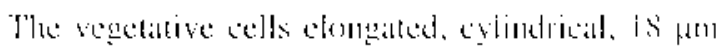

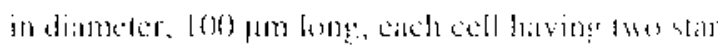

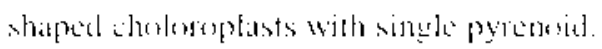

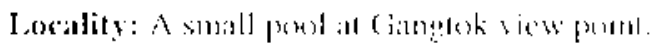

Simple No.: NISRI AI.SII 0106.

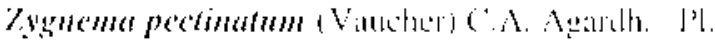
ᄀ. I I 8

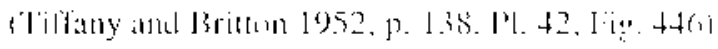

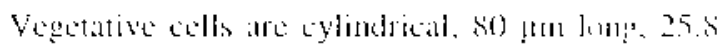

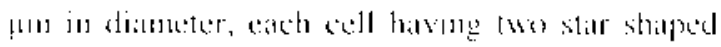

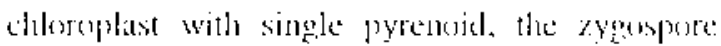

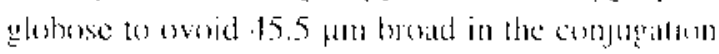
lube with median spone wall which is thick anl simusuls.

I.colity: ()n recks splatided by wated all Namthi.

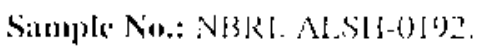

(order: l!notrichales

family: Jutrichaceac

Clothrix tentaisima ḱtety I'।. 2, Jig. 11

(kiant and (jupta 1998, p. 102, P1. 32, fije. 5)

Vegetative iclls much broader than leneth, cluadrate, 20 fur in diameter, 12.8 fm lone. chrombatophore usually in a median band with 2.4 pyrencids.

locality: $\Lambda$ small water spring at Namchi.

Sample No.: NBRI. AISH-0I86 


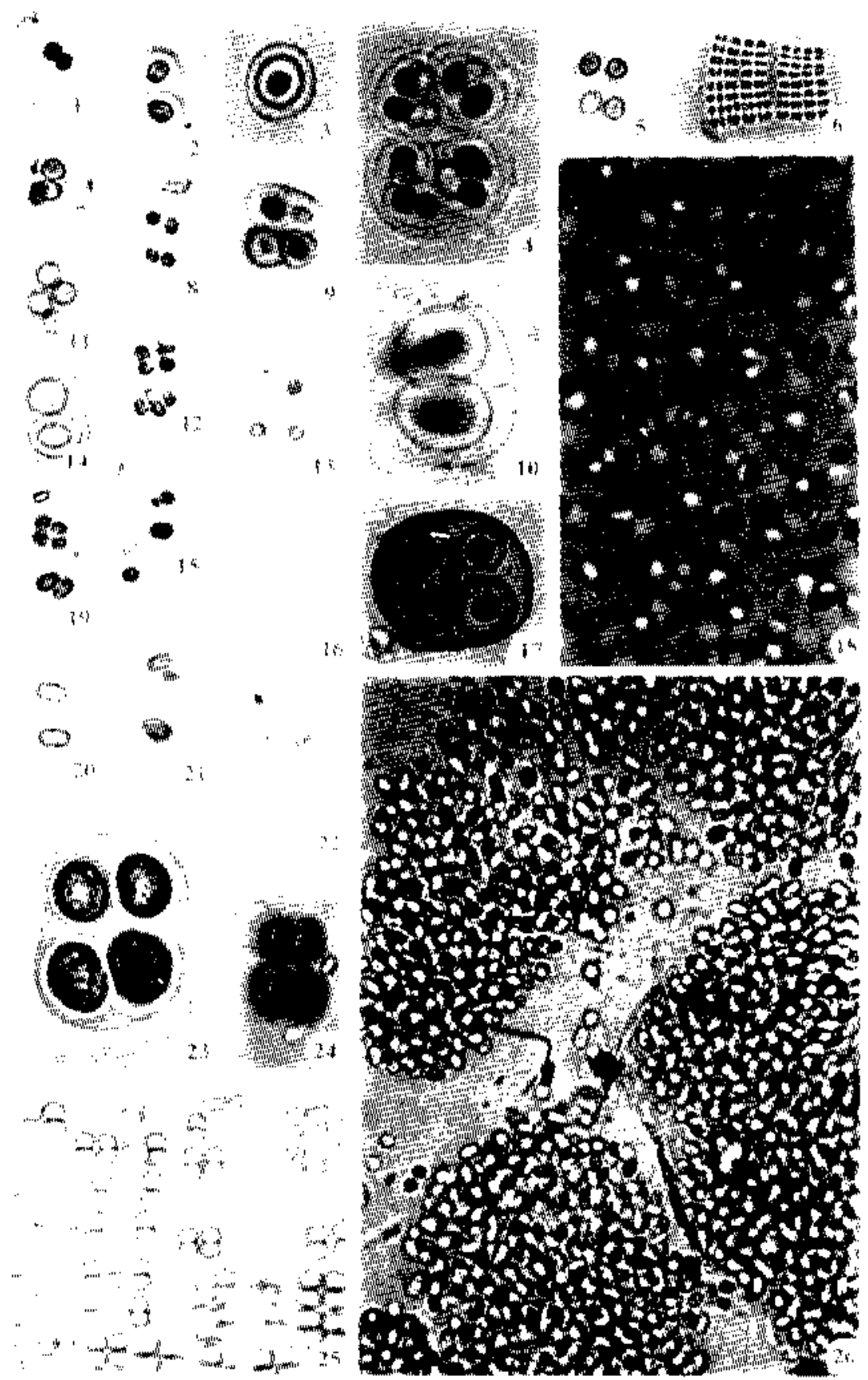

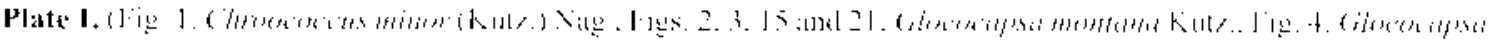

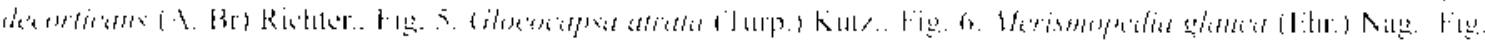

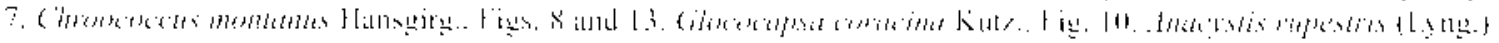

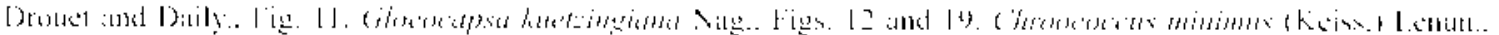

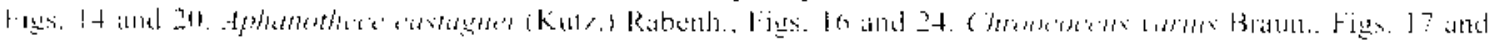

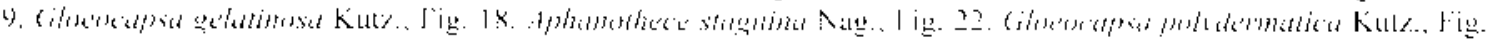

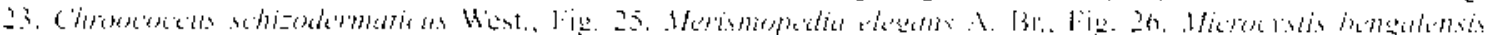
Belleriij 


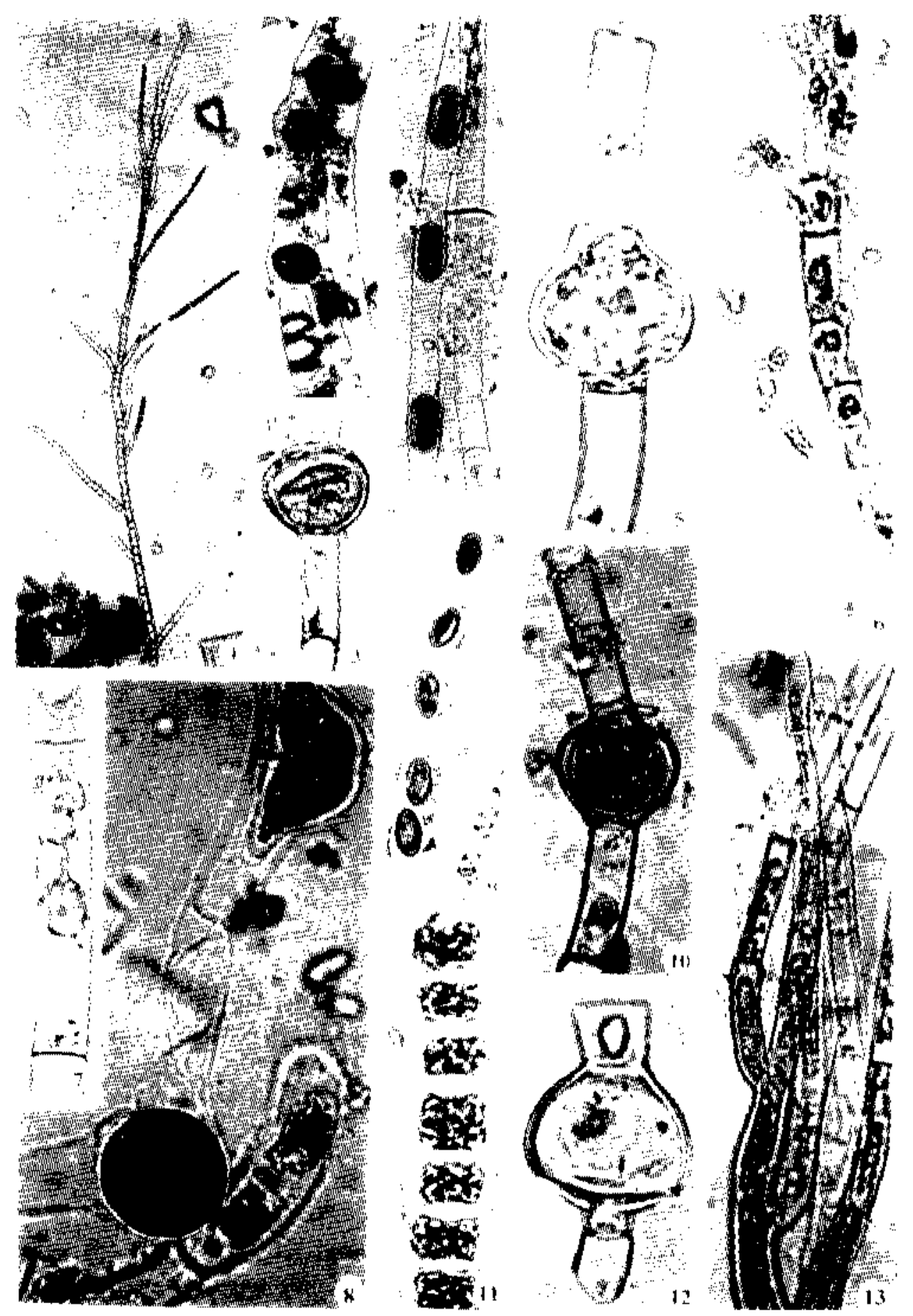

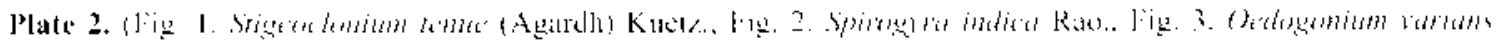

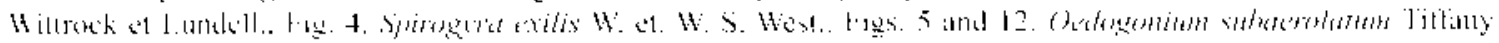

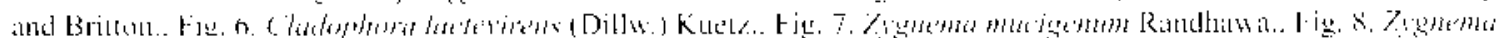

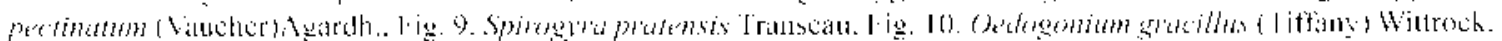

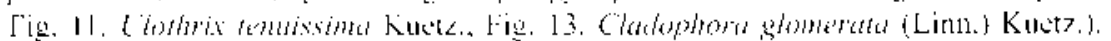




\section{RESULTS AND DISCUSSION}

During the course of the present investigation. a total of 30 taxa of two dillerent classes Cyanophycete and Chlorophyceac have been studied on the basis of morpho-taxonomic observations. Out of thirty taxa, the genus Glowerapsa, Chroococcus, Oedogonizm and Spiresyra are common in oceurrence. White, the genus Morismopertia, Aphanothere, Cladophora, Xistome, Anatystis, Microrystis, Stegindoniam and thothir aceured in dominant form. The

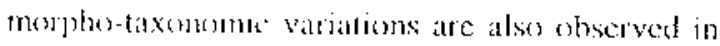
some laxis with that of esest of Indian algal l] mat. 'I his variation could be due to lhe high allitude. how temperature and obler climalic and condegical comditions of the locality.

\section{ACKNOWLLDGEMENT}

The atthors are thatukful to the Disector, Notioniald Botamial Rexearch Inslitute, lucknow

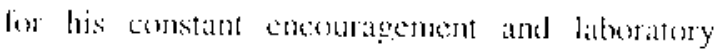
Ficilitic:s.

\section{RIEIERINCES}

Altrel. JR.B. 1978. Algal Ilora of Shillonge. I Chorephyctate. Hhees 17(1-2):33-34.

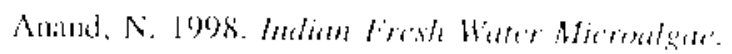

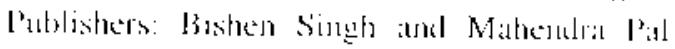

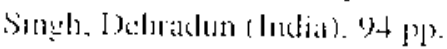

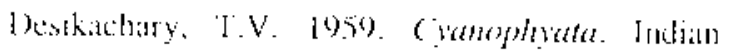

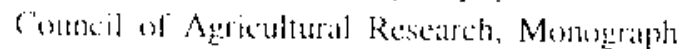

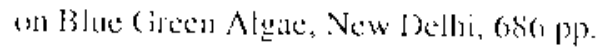

(iajt:i, R.K. 2000 . A check list of Algial 17,rat of Shillong Meghalayat Jour. Lotm Tatom. low. 26(2): $+40+4(3)$.
Kant, S. and P. Gupta. 1998. Algal fiom of Ladakh. J. Econ. Taxon. Bot Ser. 15:44I pp.

Khare, R. and M.R. Suscela. 2004. Presh water algal diversity of Nainital. Uttaranchal. India. Phytotaxonom 4: 19-24.

Prasad. B.N. and P.K. Misra. 1902. Fresh Water Algal Flora of Andaman and Nicoluar lstands. Publishers: Bishen Singh and Mahendra Pal Singh, Dehradun (Indii), $284 \mathrm{pp}$.

Prasad, B.N. and R. Mista. 1987. Destnid flural of

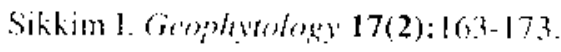

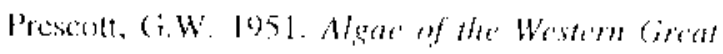

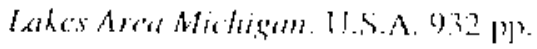

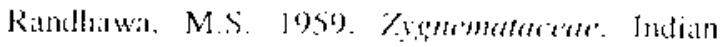
(anns il uf Agricultural Research. New Indlit. $478 \mathrm{pp}^{3}$

Raw. R.S. 1963. A buttuical koner in the Sikhien

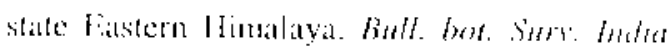
$5: 10.5204$

Sartra, SC. 1984. Algal flura of Stheim and

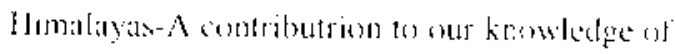

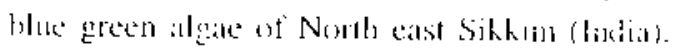
f. fire Tare Bet $4(5): 12(49)-1219$.

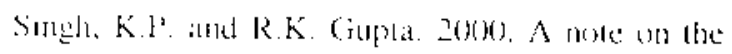

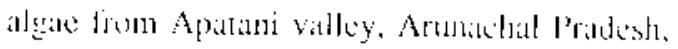
Indiat. Jent. Am. Forestry 8:38' 44

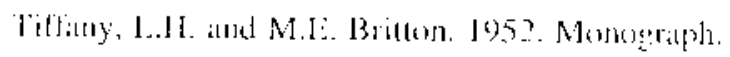

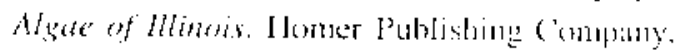
New york, 407 pp. 